\title{
Simulation of muon spin rotation for estimation of magnetic field in INO-ICAL
}

\author{
Pooja Tanty, ${ }^{a, *}$ Deepak Samuel $^{a}$ and Lakshmi P Murgod ${ }^{a}$ \\ ${ }^{a}$ Central University of Karnataka, \\ Kalaburagi, Karnataka, India \\ E-mail: tantypooja6@gmail.com, deepaksamuel@cuk.ac.in, \\ lakshmimurgod@cuk.ac.in
}

The India-based Neutrino Observatory (INO) collaboration, a multi-institutional effort to build a 50 kton magnetized Iron Calorimeter (ICAL) for studying neutrino oscillations. The magnetic field in the ICAL, induced by copper coils, will be a crucial input for the track fitting algorithms that reconstruct the four-momenta of atmospheric neutrinos. The magnetic field map as estimated from finite element techniques is usually not accurate owing to many factors including approximations in the simulation and the change in the elemental composition of the iron plates, etc. In this work, we present the results of a simulation study that employs the muon spin-rotation technique to estimate the magnetic field.

\footnotetext{
*** The European Physical Society Conference on High Energy Physics (EPS-HEP2021), *** *** 26-30 July $2021 * * *$

*** Online conference, jointly organized by Universität Hamburg and the research center DESY ***
}

${ }^{*}$ Speaker 


\section{Introduction}

INO is a mega-science project of India that will host an underground laboratory to study neutrino oscillations. This facility will house a $50 \mathrm{kton}$ magnetized Iron Calorimeter (ICAL) placed inside an underground cavern of size $132 \mathrm{~m} \times 26 \mathrm{~m} \times 20 \mathrm{~m}$ with vertical rock overburden of $1.3 \mathrm{~km}$ and at least $1 \mathrm{~km}$ of rock shielding in all directions[1]. ICAL comprises three modules each of size $16 \mathrm{~m} \times 16 \mathrm{~m} \times 14.45 \mathrm{~m}$ with a gap of $20 \mathrm{~cm}$ between them. Each module has 151 horizontal layers of iron plates interleaved with resistive plate chambers (RPCs) from which the positional and time information of hits primarliy produced by atmospheric neutrino-induced muons can be read out. The energy of neutrinos is reconstructed from the muon tracks using a track fitting algorithm. The magnetic field induced by the coils is important for the track fitting algorithm which finally decides the uncertainty in neutrino energy. Typically, the field is estimated from electromagnetic field simulation tools which are time consuming and the results may not correspond to the true magnetic field owing to changes in elemental composition. Muon spin rotation technique ( $\mu \mathrm{sr})$ is a proven tool to estimate the magnetic field through the modulations in decay curve. Previously an experimental study was performed with the prototype detectors the results of which were promising. In this work, to understand the effects of the time resolution and muon polarization, a Geant4-based simulation framework for muon spin rotation was developed and the results of the achievable accuracy of the magnetic field as a function of parameters like muon polarization and time resolution are presented.

\section{Simulation Setup}

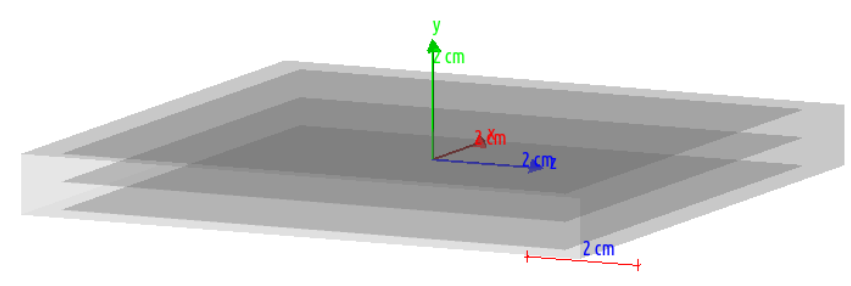

Figure 1: Prototype detector setup simulated in GEANT4

To measure the magnetic field inside a material using the $\mu \mathrm{sr}$ technique, we need a polarized source of muons and a detector to measure the timing distribution of decay electrons over a dynamic range of at least 5 lifetimes, depending on the magnetic field. Figure 1 shows the prototype detector setup consists of an iron plate of dimensions $90 \mathrm{~mm} \times 90 \mathrm{~mm} \times 0.1 \mathrm{~mm}$ simulated in Geant 4 . The iron plate is sandwiched between two RPCs of the same specifications as the iron plate. A gap of $4.6 \mathrm{~mm}$ is maintained between the iron plate and the RPC. The simulation aims to test the feasibility of using cosmic muons, which have a polarization of about $30 \%$, to observe the $\mu$ sr signal. 


\section{Muon spin rotation}

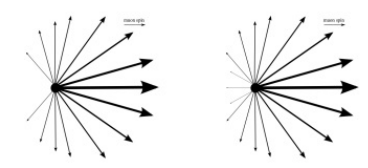

Figure 2: Left: The angular distribution for maximum energy positrons. Right: The distribution for all energies. The muon spin points towards the right.

The decay of $\mu^{+/-}$gives out $e^{+/-}$and (anti) neutrinos. As a result of parity violation, the positron/electron prefers to be emitted in (opposite) to the direction of muon spin as shown in figure 2. Experimentally it has been shown that the polarization of cosmic muons to be around 26\%[3].

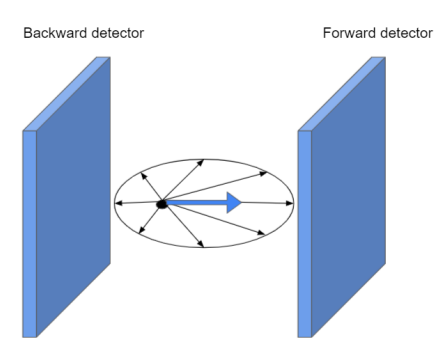

Figure 3: The muon precessing in transverse magnetic field with the preferential direction of $e^{+}$.

When the polarized muon is placed in a magnetic field, the spin vector of the muon starts precessing about the axis of the magnetic field with the Larmor frequency. The emission rate of $e^{+} / e^{-}$in a given direction will now depend on muon polarization $\mathrm{P}$ and the oscillation frequency (Larmor frequency)[4]:

$$
d N(t)=N_{0}\left(e^{-} \frac{t}{\tau_{\mu}}\right)\left[1+P(t)_{x} \cos (\omega t+\phi)\right] d t d \sigma
$$

where $\omega$ is the larmor frequency which is $135 \mathrm{MHz} / \mathrm{T}$ for muons.
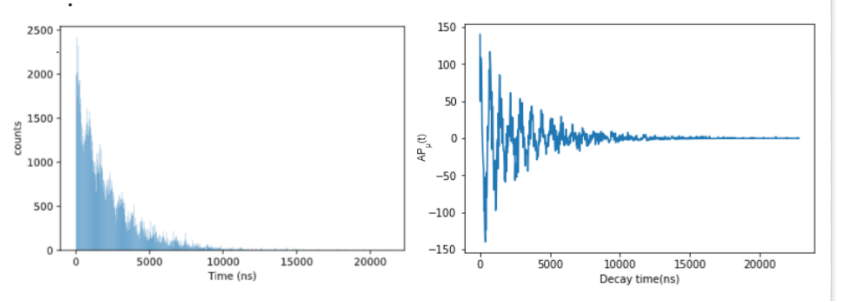

Figure 4: $\mu$ sr signal after subtracting exponential component from decay spectrum.

In an experiment, from the knowledge of $d N(t)$ and the polarization $\mathrm{P}$ and muon lifetime $\tau$, the magnetic field $B_{l o c}$ can be estimated:

$$
\omega=\gamma B_{l o c}
$$


a)

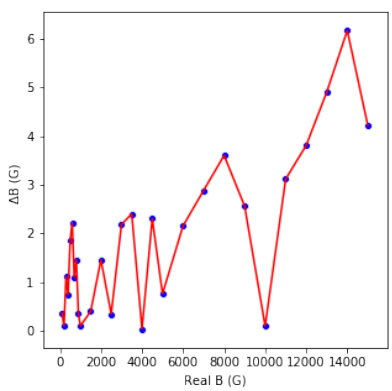

b)

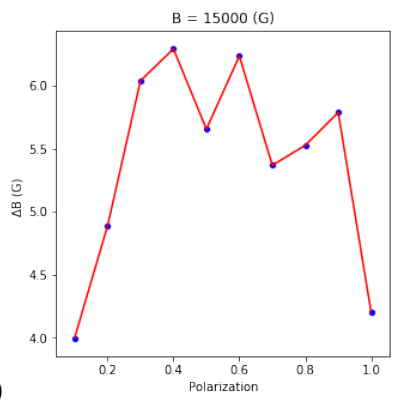

c)

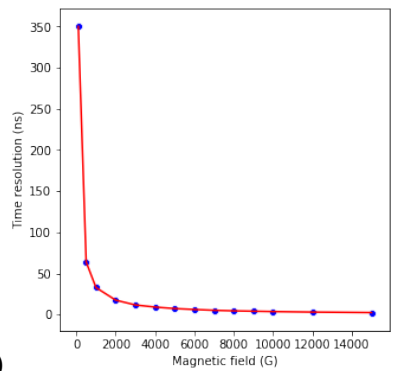

Figure 5: (a) Difference in estimated and real magnetic field, (b) Effect of muon polarization in field estimation, (c) Estimated magnetic field for different time resolution

The $\mu$ sr signals are obtained after removing the exponential component from the timing spectrum as shown in figure 4.

\section{Results and Performance}

The results from the simulations are promising and are shown in figure 5. While the effect of muon polarization and magnetic field seem to be marginal, the effect of time resolution seems to be the deciding factor. We are still improving the performance of the code developed to introduced the effect of spatial resolution which may deteriorate the quality of the results.

\section{Conclusion}

In this work, we have developed a Geant4-based simulation tool to study the feasibility of using cosmic muons, to gauge the magnetic field inside an iron plate. While the first results seem to be promising, the effects of spatial resolution and detector noise needs further investigation.

\section{References}

[1] Meghna K K, Performance of RPC detectors and study of muons with the Iron Calorimeter detector at INO, 2015.

[2] S N Sen Gupta and M S Sinha, Decay asymmetry of cosmic ray muons, In:Proceedings of the Physical Society 79.6 (1962), pp. 1183-1196.

[3] Satio Hayakawa, Polarization of cosmic-ray $\mu$ mesons: Theory Phys. Rev. 108,1533,1957.

[4] Alex Amato. Physics with muons: From atomic physics to solid state physics, 2019.

[5] N.Panchal, An efficient cosmic muon veto detector for experiments at shallow depth and feasibility of internal magnetic field measurements using cosmic muons, 2019. 